\title{
Towards a systematic approach to resource optimization management in the healthcare domain
}

\author{
$\underline{\text { P.A. Khaiter }}^{\text {a }}$, M.G. Erechtchoukova ${ }^{\text {a }}$ and Y.A. Aschane ${ }^{\text {a }}$ \\ ${ }^{a}$ School of Information Technology, Faculty of Liberal Arts and Professional Studies, York University, \\ Toronto, Canada \\ Email:pkhaiter@yorku.ca
}

\begin{abstract}
Increasing patient demand, constrained physical resources, and a rising cost of operations are imperative concerns in healthcare management which require improvements to the way medical services are provided to the public. The urgency of the problem in Ontario, Canada has forced the Provincial Government to put a plan in place to increase access and reduce wait times for major health services including cancer surgery, cardiac procedures, cataract surgery, hip and knee replacements, general surgery, paediatric surgery, and MRI and CT exams (Ontario, 2008). The main directives of the plan include four goals:
\end{abstract}

- significantly increase the number of procedures to reduce the backlog that has developed over the last decade;

- invest in new, more efficient, technology such as MRI machines and provide longer hours of their operation;

- standardize best practices for both medical and administrative functions to improve patient flow and efficiency; and

- collect and report accurate and up-to-date data on wait times to allow better decision making and increase accountability.

In addition, Ontario's health budget for 2017-18 will grow by $\$ 1.6$ billion, aiming to reduce wait times and ease hospital overcrowding, including program funding for hospitals to be boosted by 3 per cent, or $\$ 518$ million, as compared to the last year (Boyle, 2017). To achieve the targets, healthcare management at different levels of decision-making should plan effectively to reduce costs, maximize profit, and increase the quality of their services by undergoing a thorough investigation on resource planning, scheduling, and utilization. In this paper, we argue for a systematic approach to resource management in the healthcare domain, focusing on the problem of scheduling medical procedures at the departmental level on the basis of information technology and applicable optimization techniques. The approach is to follow a seven-step exercise itemized in point form:

- a thorough study of the flow of operations and procedures rendered by the healthcare provider;

- identification of all of the actors involved in the operations and management decisions to be made at each hierarchal level (i.e., strategic, tactical, and operational);

- determination of different patient types served by a given provider because their characteristics are important for the selection of optimization methods;

- deciding on the set of applicable performance indicators;

- selecting the most suitable for the task at hand scheduling variables and scheduling types (i.e., in block of resources, open scheduling, allocation or advanced scheduling);

- incorporating uncertainty characteristics of various actors and operations;

- on the basis of the above factors, selecting and applying optimization method(s) or a certain combination thereof, satisfying specific features of the problem at hand in the best way.

Operations of the Image Guided Therapy (IGT) Department of the Hospital for Sick Children ("SickKids"), Toronto, Canada have been taken as a sample object in the study. The IGT department provides valuable diagnostic and therapeutic data using procedures that involve different forms of anesthesia or sedation administered to the patients (Khaiter et al., 2015). The study demonstrated that none of the investigated optimization algorithms was able to minimize the IGT schedules with regard to all selected time-based performance criteria. Each algorithm generated schedules which are more efficient from the perspective of a single performance indicator, but not optimal for the others. It is reasonable to assume that specific features of the IGT department (i.e., multi-server environment and variable-length blocks) make the optimization of heir scheduling a complex non-trivial problem requiring a hybrid approach that combines several optimization techniques.

Keywords: Healthcare domain, patient type, scheduling variable, performance indicators, optimization 


\section{INTRODUCTION}

The management aspect of healthcare is an indispensable component of the field, still seeking significant improvements to provide quality and effective services to patients. While facing a growing demand for, and rising costs of medical services, quality management in the healthcare domain is gaining significant attention (Guinet and Chaabane, 2003). This requires that management at different levels of decision-making (i.e., strategic, tactical, and operational) acts effectively to reduce costs, maximize profit, and increase the quality of services by undergoing a systematic investigation on resource planning, scheduling, and utilization. The urgency of the problem in Ontario, Canada has forced the Provincial Government to put a plan in place to increase access and reduce wait times for major health services including cancer surgery, cardiac procedures, cataract surgery, hip and knee replacements, general surgery, paediatric surgery, and MRI and CT exams (Ontario, 2008). This strategy has since been expanded to include all surgeries and time spent in emergency rooms. The paper presents a systematic approach to resource management in the healthcare domain, focusing on the problem of scheduling medical procedures at the departmental level on the basis of information technology and applicable optimization techniques. The approach has been applied to the problem of efficient scheduling in a multi-server dynamic medical environment and for selected time-based performance criteria.

\section{SYSTEMATIC APPROACH TO RESOURCE MANAGEMENT}

The task of improving the current practices of scheduling in a medical department can be approached by applying information technology and formal methods of optimization, including a review of all possible internal and external entities which may affect the performance of the core system under the study.

This is analogous Albert Einstein's claim that, "if I were given one hour to save the planet, I would spend 59 minutes defining the problem and one minute resolving it." This idea is often overlooked by most organizations, who do not spend enough time on understanding the problem when launching new projects. The same is true in the healthcare domain, which demands rigorous definition of the problems and articulation of all possible factors and variables which may affect the system being studied and change expected outcomes. Accordingly, we discuss possible variables, factors, and scenarios in view of their potential influence on the healthcare domain and related optimization approaches.

\subsection{Decision level impact analysis}

Performance in healthcare is affected by decisions made at different management levels. These involve external and internal policies and rules designated to outline how various pertinent resources are assigned, controlled, and coordinated and may range from setting goals for the entire healthcare domain to specific decisions regarding day-to-day activities of departments and medical centers. Some of these goals may be short-term, while others may have long-term implications on the domain. From these points of view, decisions in the healthcare domain can be broadly classified into three hierarchal levels: strategic, tactical, and operational (Hans et al., 2012). The operational level can be further categorized into offline (i.e., advanced) and online (i.e., real-time reactive) decision-making (Hulshof et al., 2012). This hierarchy is displayed in Figure 1.

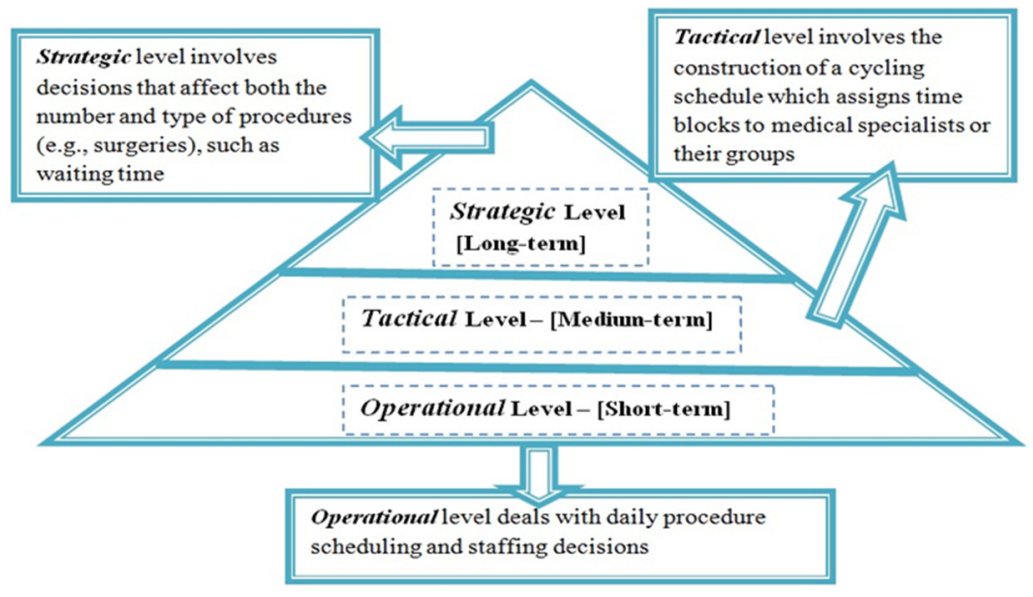

Figure 1. The hierarchy of decision levels in healthcare. 
Khaiter et al., Towards a systematic approach to resource optimization management in the healthcare domain

\subsection{Actors in healthcare systems}

A fundamental step in addressing the problem of resource management is the identification of actors (i.e., external agents or person-roles) outside the system that directly interact with the system by supplying or receiving information. For each category of actors, there are corresponding use cases or activities performed by the system in response to their requests. The overall flow of control during the use case execution is modelled by activity diagrams whereas realization of the use cases (i.e., determination of collaborating objects and messages they send to each other to carry out the use case) is done by sequence diagrams (see Khaiter et al., 2015 for details). Doctors, nurses and patients are among the most important actors in any healthcare system.

\subsection{Patient types and their characteristics}

Patients can be divided in two large categories: elective patients and non-elective patients. Those patients for whom their procedure can be planned in advance form the elective class, whereas those for whom the procedure is unplanned or unexpected and requires an integration into the existing schedule belong to the non-elective class (Samudra et al., 2016). In turn, the elective class includes inpatients who are admitted to the hospital and outpatient patients who do not require hospital admission, whereas the non-elective class combines emergency patients, for whom procedure should be performed immediately, and urgent patients whose procedure can be postponed for a short time (Adan et al., 2011; Beliën et al., 2006; Ferrand et al., 2010).

\subsection{Performance measures}

In their review, Cayirli and Veral (2003) noted a variety of healthcare performance criteria used in the literature. Table 1 gives a summary of a limited set of the performance indicators relevant to the present study.

Table 1. Performance measures in healthcare

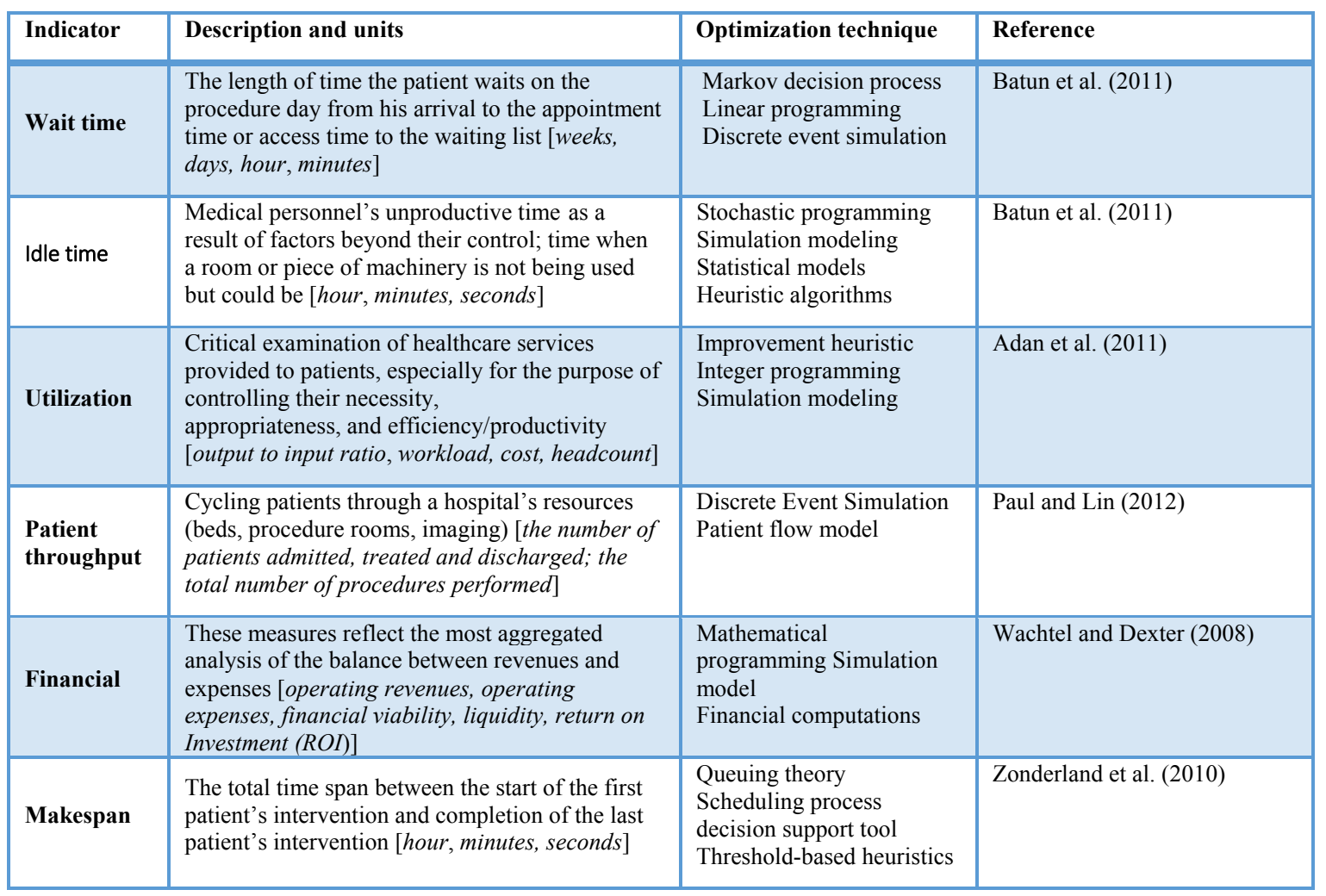

\subsection{Scheduling variables, types and perspectives}

Scheduling requires identification of variables, which, in turn, depend on a type of a requested procedure and necessary resources. The following variables can be used at the departmental level: room, day, time, or an amount of capacity (with regard to beds, operation rooms, recovery time, etc.) and in different combinations and ways. The latter includes blocks of resources (i.e., intervention rooms or a day) that are assigned to a medical practitioner or their group (e.g., cardiology), and they are only allowed to book cases into these blocks, 
open scheduling, whereby practitioners can plan procedures into an arbitrary intervention room with no restriction (van Oostrum et al., 2010) as well as allocation scheduling (i.e., a process of fixing patient-to-roomand-time and determining the operation rooms starting times and intervention sequence on the planned day) or advance scheduling, whereby a patient is assigned an intervention date, and it can be done either in a static or dynamic way. The former assumes that patients arrive one by one on a fixed intervention date/time with minimum delay while the latter assumes batch input put onto a waiting list (Magerlein \& Martin, 1978). There are three perspectives to consider: (1) the patient level, whereby the decision variables are formulated based on individual patients or patient type; patients are scheduled in two stages: first, patients are assigned to days and rooms and next, the exact daily intervention sequencing (i.e. time aspect) is determined (Fei et al., 2010); (2) the discipline level, in which a decision is made for a whole department or division of a medical specialty (e.g. Paediatrics) (Adan et al., 2011); and (3) the medical practitioner level, which involves surgeons who can define the intervention blocks and then be assigned to intervention rooms (Denton et al., 2010).

\subsection{Uncertainty aspect}

The operations of healthcare systems are highly stochastic. It is difficult to have precise intervention planning and scheduling outcomes because of the uncertainty associated with the characteristics of various actors involved in the healthcare domain. For example, the arrival time uncertainty of non-elective patients, and the duration of the procedure itself, which is made difficult to forecast because of artificial delays or natural personal characteristics of the patient or level of expertise of individual servers or their teams.

\section{IMAGE GUIDED THERAPY (IGT) DEPARTMENT}

Operations of the Image Guided Therapy (IGT) Department of the Hospital for Sick Children ("SickKids"), Toronto, Canada have been taken as a sample object in the study. The IGT department provides valuable diagnostic and therapeutic data using procedures that involve different forms of anesthesia or sedation administered to the patients. Procedures done at the IGT department can be divided into three major groups: (1) general anesthetic (GA) cases, which require complete anesthesia of the patient; (2) registered nurse (RN)sedation or non-GA cases, which do not require complete anesthesia; and (3) local cases done under local anesthesia (Khaiter et al., 2015). There are also different types of imaging produced: ultrasound, fluoroscopy/digital subtraction angiography and computerized axial tomography (CT/CAT scan). The IGT department involves interactions of various groups of actors as shown in Figure 2.

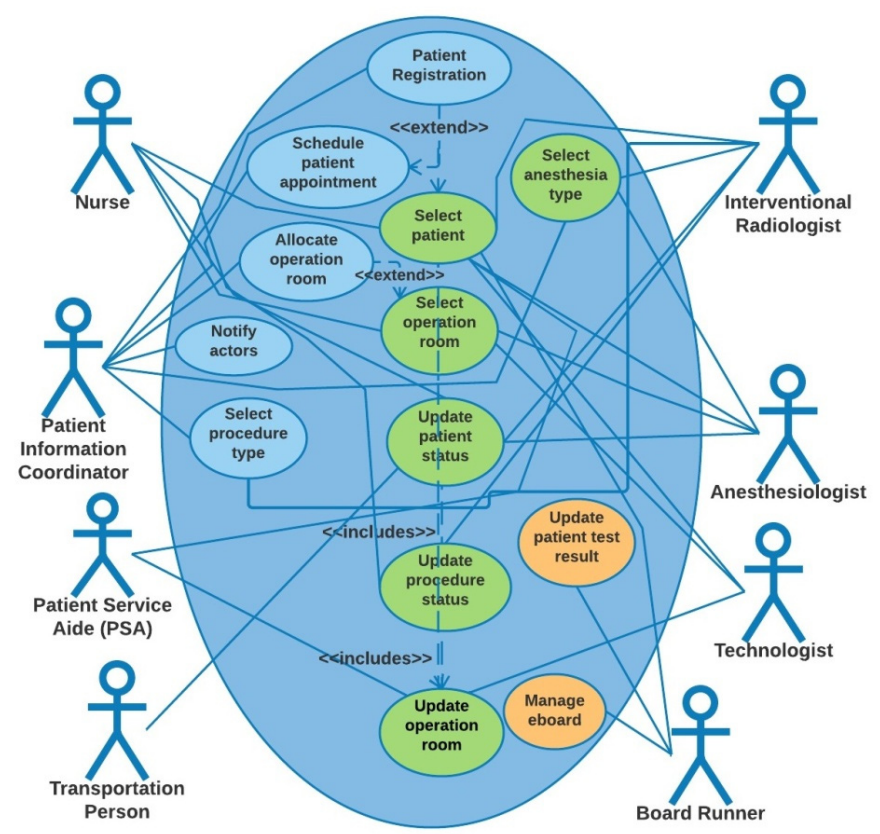

Figure 2. UML use case diagram presenting actors and functionality of the IGT Department.

The IGT department deals with two classes of patients: (1) elective patients (which can be either inpatient or outpatient) and (2) non-elective patients (e.g., emergency patients requiring immediate diagnostics and treatment) (see also Table 2 for details). The type of required procedure is determined by a patient's requisition 
Khaiter et al., Towards a systematic approach to resource optimization management in the healthcare domain

form, which is the starting point in the flow of operations. Once the requisition form is assessed and accepted by the department, the process of scheduling and resource assignment begins. Figure 3 presents a UML activity diagram for the elective patient requisition assessment and resource assignment process.

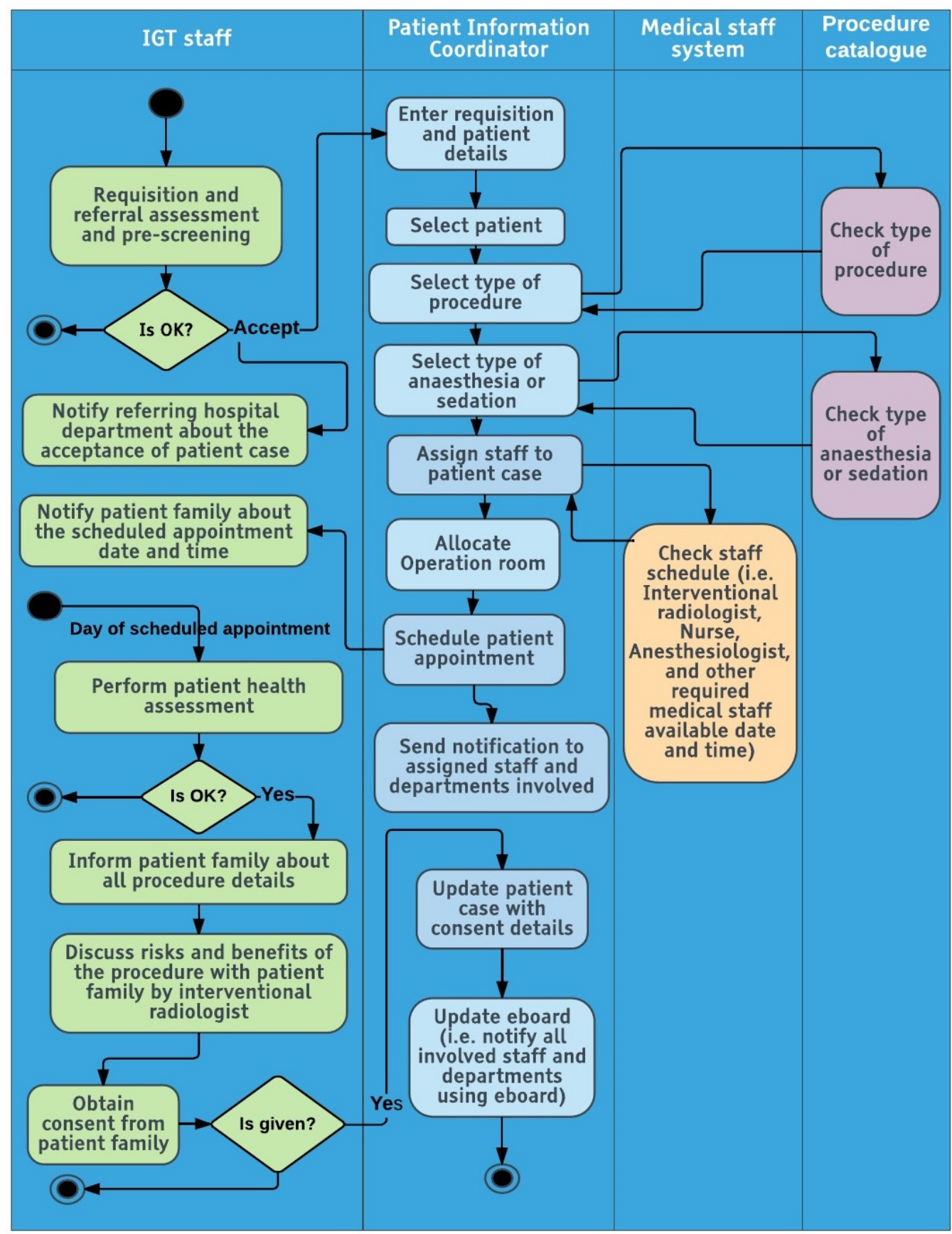

Figure 3. UML Activity diagram for elective patient requisition assessment and resource assignment process.

\section{OPTIMIZATION METHODS IN A SYSTEMATIC APPROACH}

Optimization methods are applied to analyze the complexity of system inputs, processes and outcomes with specific constraints to provide the best possible solution for a given problem. There are wide ranges of methodologies which are in the domain of operations research that arrive at optimal or near-optimal solutions to complex decision-making problems (Cardoen et al., 2010). Figure 4 presents a summary of optimization techniques commonly used in healthcare systems. 


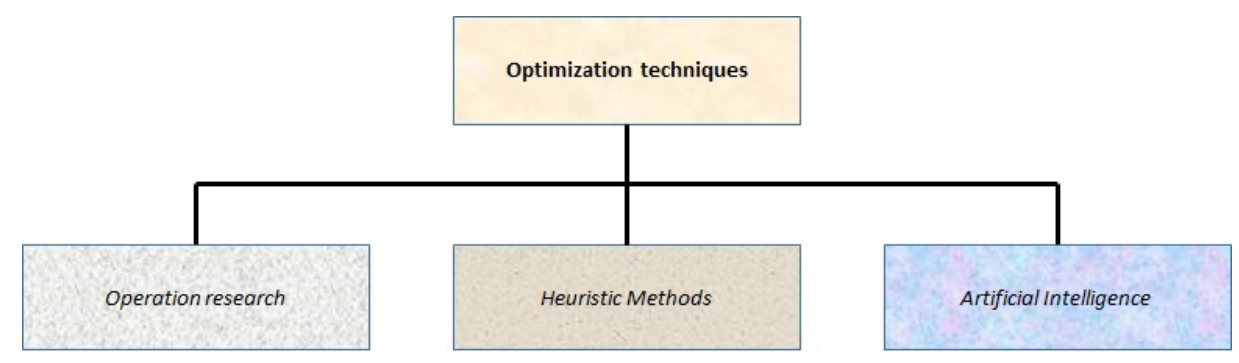

Figure 4. Optimization techniques in healthcare.

Specific details and mathematical articulations of these techniques largely depend on a particular case. The systemic approach has been applied to the problem of optimal scheduling in the IGT department. A scheduling unit in the department is an appointment which is characterized by planned start time, actual start time, planned end time and actual end time, depending on the complexity of a required interventional procedure. A heuristic scheduling process used in the department follows "first-come, first-served" (FCFS) principle provided there is an available time slot on a given day. A preliminary static schedule is available at the beginning of the day for two categories of patients (i.e., outpatients and inpatients). A dynamic change to the daily schedule is made as the necessity arises during the day, and it may affect any type of patients. The appearance of emergency cases causes the adjustment of the daily schedule. Several optimization techniques have been investigated in the study regarding such time-based performance measures as patient wait time, idle time of doctors and nurses; idle and turnover time of equipment and staff overtime. However, the complexity of the IGT environment and the diversity of procedures performed by the staff do not make the departmental operations fully suitable for the case as the optimization could not be achieved for all performance indicators concurrently, and an optimal schedule should somehow compromise them.

\section{DISCUSSION AND CONCLUSIONS}

The systematic approach presented in this paper is based on a thorough study of the flow of operations of the healthcare provider and identification of all of the actors involved in the operations, and management decisions to be made at each hierarchal level (i.e., strategic, tactical, and operational). Different patient types and their characteristics need to be taken into account, especially when selecting the set of performance measures and scheduling variables most suitable for the problem at hand. It is also desirable to have uncertainty characteristics of various actors and operations incorporated into the scheduling algorithms.

The IGT department deals with all categories of patients listed in sub-section 2.3 and applies different types of scheduling (i.e., in blocks of resources and open, allocation and advance). Their scheduling decisions can be viewed from each of the three perspectives (i.e., patient, discipline and medical practitioner). As the study demonstrated, none of the investigated optimization algorithms was able to minimize the schedules with regard to all selected time-based performance criteria. Each algorithm generated schedules which are more efficient from the perspective of a single performance indicator and, as such, can be more preferable for different actors: departmental or hospital administration, patients or medical staff. It is possible to assume that specific features of the IGT (i.e., multi-server environment and variable-length blocks) make the optimization of resource management in the department a complex non-trivial problem which most likely cannot be solved by a single algorithm and calls for a hybrid approach combining several optimization techniques. This issue will be a topic for our future endeavours.

\section{ACKNOWLEDGMENTS}

We are grateful to the entire IGT team at the Hospital for Sick Children for providing information on the operations of the department, as well as for their contributions to this project. We express our special thanks to Dr. Bairbre Connolly, Dr. Albert Aziza and Dr. Afsaneh Amirabadi for their availability, valuable clarifications, discussions and gracious support. The authors are thankful to editors and anonymous reviewers for their thoughtful suggestions and helpful comments on the manuscript.

\section{REFERENCES}

Adan, I., Bekkers, J., Dellaert, N., Jeunet, J., and Vissers, J. (2011). Improving operational effectiveness of tactical master plans for emergency and elective patients under stochastic demand and capacitated resources. European Journal of Operational Research, 213(1), 290-308. 
Khaiter et al., Towards a systematic approach to resource optimization management in the healthcare domain

Batun, S., Denton, B. T., Huschka, T. R., and Schaefer, A. J. (2011). Operating room pooling and parallel surgery processing under uncertainty. INFORMS Journal on Computing, 23(2), 220-237.

Beliën, J., Demeulemeester, E., and Cardoen, B. (2006). Visualizing the demand for various resources as a function of the master surgery schedule: A case study. Journal of Medical Systems, 30(5), 343-350.

Boyle, T. (2017). \$1.6 billion in health money aims to reduce wait times, ease overcrowding. Toronto Star, April 27, 2017. www.thestar.com/news/canada/2017/04/27/16-billion-in-health-money-aims-to-reducewait-times-ease-overcrowding.html (accessed July 31, 2017).

Bruni, M. E., Beraldi, P., and Conforti, D. (2015). A stochastic programming approach for operating theatre scheduling under uncertainty. IMA Journal of Management Mathematics, 26(1), 99-119.

Cardoen, B., Demeulemeester, E., and Beliën, J. (2010). Operating room planning and scheduling: A literature review. European Journal of Operational Research, 201(3), 921-932.

Cayirli, T., and Veral, E. (2003). Outpatient scheduling in health care: a review of the literature. Production and operations management, 12(4), 519-549.

Denton, B. T., Miller, A. J., Balasubramanian, H. J., Huschka, T. R. (2010). Optimal allocation of surgery blocks to operating rooms under uncertainty. Operations Research, 58(4), 802-816.

Fei, H., Meskens, N., and Chu, C. (2010). A planning and scheduling problem for an operating theatre using an open scheduling strategy. Computers and Industrial Engineering, 58(2), 221-230.

Ferrand, Y., Magazine, M., and Rao, U. (2010). Comparing two operating-room-allocation policies for elective and emergency surgeries. In: Proceedings of the 2010 Winter Simulation Conference (WSC), (B. Johansson, S. Jain, J. Montoya-Torres, J. Hugan, and E. Yucesan - Eds.). Baltimore, Maryland, USA, 5-8 December, pp. 2364-2374.

Guinet, A., and Chaabane, S. (2003). Operating theatre planning. Int. J. Production Economics, 85(1), 69-81.

Hans, E.W., van. Houndenhoven, M., and Hulshof, P.J.H. (2012). A framework for health care planning and control. In: Handbook of healthcare system scheduling (R. Hall - Ed.), Springer, New York, pp. 303-320.

Hulshof, P.J.H., Kortbeek, N., Boucherie, R.J., Erwin W., and Hans, E.W. (2012). Taxonomic classification of planning decisions in health care: a structured review of the state of the art in OR/MS. Health Systems, 1(2), $129-175$.

Khaiter, P. A., Erechtchoukova, M. G., Connolly, B., Aziza, A., Ahmed, K. S., Khaiter, D., and Maclellan, B. (2015). Developing an eBoard for resource management in the Image Guided Therapy Department. In: MODSIM 201521 st International Congress on Modelling and Simulation, (Weber, T., McPhee, M.J. and Anderssen, R.S. - Eds), Modelling and Simulation Society of Australia and New Zealand, November 29 December 4, pp. 1654-1660.

Magerlein, J. M., and Martin, J. B. (1978). Surgical demand scheduling: a review. Health Services Research, $13(4), 418-433$.

Min, D., and Yih, Y. (2010). An elective surgery scheduling problem considering patient priority. Computers and Operations Research, 37(6), 1091-1099.

Ontario Ministry of Health and Long-term Care. (2008). Ontario wait trimes. www.health.gov.on.ca/en/public/programs/waittimes/default.aspx (accessed July 31, 2017).

Paul, J. A., and Lin, L. (2012). Models for improving patient throughput and waiting at hospital emergency departments. The Journal of emergency medicine, 43(6), 1119-1126.

Samudra, M., Van Riet, C., Demeulemeester, E., Cardoen, B., Vansteenkiste, N., and Rademakers, F. (2016). Scheduling operating rooms: achievements, challenges and pitfalls. J. Sched, 19(5), 493-525.

van Oostrum, J. M., Bredenhoff, E., and Hans, E. W. (2010). Suitability and managerial implications of a Master Surgical Scheduling approach. Annals of Operations Research, 178(1), 91-104.

Wachtel, R. E., and Dexter, F. (2008). Tactical increases in operating room block time for capacity planning should not be based on utilization. Anesthesia and Analgesia, 106(1), 215-226.

Zonderland, M. E., Boucherie, R. J., Litvak, N., and Vleggeert-Lankamp, C. L. A. M. (2010). Planning and scheduling of semi-urgent surgeries. Health Care Management Science, 13(3), 256-267. 